\title{
Vestibular sway: Parameters of the eliciting stimulus
}

C. DENTON FERHALD ${ }^{1}$ AND JOHN W. MOORE

UNIVERSITY OF MASSACHUSETTS, AMHERST
Postural sway was elicited in human $S$ s using various combinations of low frequency-low amplitude sinusoidal electrical stimulation at the mastoid processes. Amount of sway was a $V$-shaped function of the stimulus frequency at low amplitude and an inverted $V$ function at the higher amplitude. Amplitude-frequency combinations optimal for eliciting overall sway were different from those most suitable for use in conditioning.

Recent experiments have indicated that low frequency sinusoidal electrical stimulation of low amplitude applied to the human vestibular apparatus elicits postural sway and that this response can be conditioned (Revusky et al, 1965). Dzendolet (1963) found that thresholds of vestibular sway depend upon stimulus frequency. The present experiment investigated the joint effects of stimulus frequency and amplitude in the context of a classical conditioning situation.

\section{Method}

Postural sway was recorded by having the $\mathrm{S}$ stand on a low platform supported by a steel fulcrum and two steel bars. Lateral sway exerted force on one underlying steel bar while withdrawing force from the contralateral bar. Strain gages affixed to these bars formed two legs of a Wheatstone bridge, the output of which provided a continuous oscillographic record of lateral sway. The platform was quite stable and provided little sensation of rocking. The apparatus and general procedure are described in greater detail in Revusky et al (1965).

Ss were blindfolded and remained standing on the platform for the entire experimental session. They were instructed to maintain a standard posture: feet together, knees relaxed, equal weight on both legs, and hands clasped in front. The purpose of the experiment was described as "recording brain waves to sounds under mild stress." These instructions presumably explained the presence of the fluid electrodes on the Ss' mastoid processes and the requirement of standing for a long period of time, while masking the true purpose of the experiment. Each $\mathrm{S}$ received 3 initial trials consisting of presentation of the conditioned stimulus (CS) alone. This was a $1000 \mathrm{cps}$ tone of $70 \mathrm{db}$ SPL and of $5.5 \mathrm{sec}$.duration. These trials were followed by 8 blocks of trials which, for conditioning groups, consisted of 3 trials on which the CS was paired with the unconditioned stimulus (UCS) followed by a test trial consisting of presentation of the CS alone. The CS-UCS interval was a constant .5 sec. on conditioning trials. The UCS was a 5 sec. sinusoidal electrical stimulus of either $.2, .5$, and $1.0 \mathrm{cps}$ at either high or low peak amplitudes of .27 and .12 milliamperes. These levels of stimulation are too low to seriously threaten the S's equilibrium, and, in fact, most Ss remained unaware of their swaying or the UCS.

Twelve male undergraduate volunteers were randomly assigned to each combination of UCS amplitude and frequency, thereby completing a 2 by 3 factorial design. A control group, comprised of an additional $12 \mathrm{Ss}$, received the same number of CS presentations as the conditioning groups, but the UCS was never presented. The intertrial interval was a constant $70 \mathrm{sec} .$, and all Ss were allowed a brief period halfway between each trial to stretch and readjust their posture. A continuous white masking noise of $70 \mathrm{db}$ SPL was on for the entire experimental session.

Response amplitude was measured as the difference between the greatest positive and negative excursions of the oscillographic recording pen within a specified scoring interval. There were two scoring intervals for each trial: a pre-trial score was obtained from the $5.5 \mathrm{sec}$. interval immediately before CS-onset and an on-trial score was obtained from the $5.5 \mathrm{sec}$. while the CS (or CS plus UCS) was on.

\section{Results and Discussion}

Analysis of variance of amplitude scores showed that the six conditioning groups did not differ over the 3 initial test trials. Furthermore, all groups showed a gradual increase in both pre- and on-trial response amplitude over trials which was likely due to cumulative fatigue. The difference in response amplitude between the first and second halves of the experiment was significant, $F(1,66)=39.92, p<.001$.

Figure 1 shows the mean response amplitude for each group pooled over trials. ${ }^{2}$ With the lower amplitude

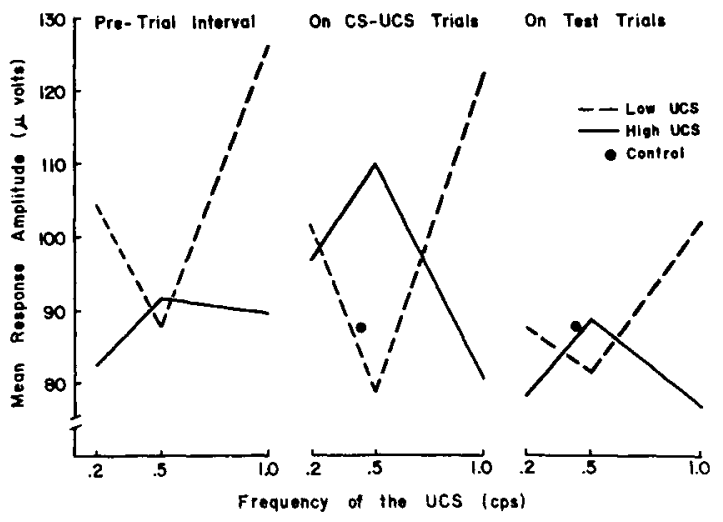

Fig. 1. Mean response amplitude for each group during pre-trial interval, on conditioning (CS-7CS) trials, and on test (CS-alone) trials. 
UCS, both pre- and on-trial sway was a V-shaped function of UCS frequency. The rank order of frequencies in terms of response amplitude was $1.0, .2$, and $.5 \mathrm{cps}$ from greatest to least. Increasing the amplitude of the UCS produced a complete reversal of this rank order, with sway to 1.0 and .2 cps greatly suppressed while that to .5 cps was enhanced, especially during the on-trial intervals. This pattern suggests (a) that the vestibular apparatus is more sensitive to frequencies of .2 and 1.0 than $.5 \mathrm{cps}$, a conclusion which agrees with the thresholds for vestibular stimulation at various frequencies determined by Dzendolet (1963), and (b) that excessive vestibular stimulation engenders compensatory mechanisms which inhibit postural sway. Presumably, then, further increases in the amplitude of a .5 cps stimulus would also have resulted in suppression of sway.

Figure 2 is a plot of the mean differences in response amplitude between pre- and on-trial intervals . Positive

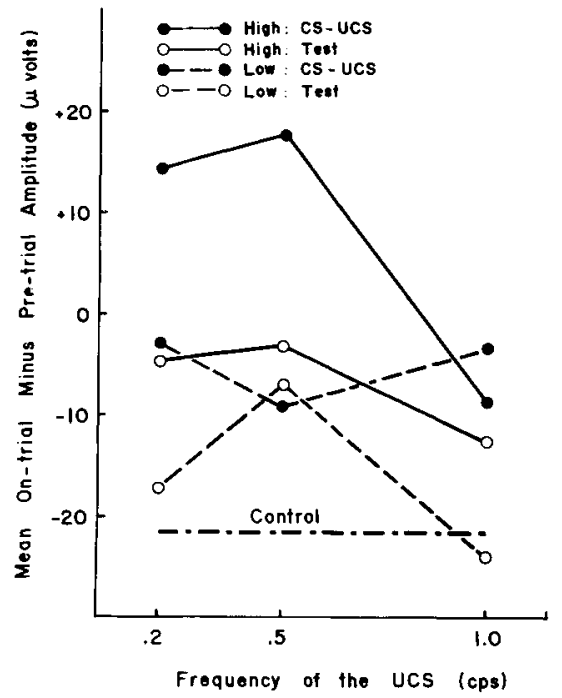

Fig. 2. Mean amplitude difference (on-trial amplitude minus pretrial amplitude) for conditioning (CS-UCS) trials and test (CSalone) trials for each group. scores indicate that on-trial amplitude exceeded the pre-trial level. Figure 2 shows that the CS tended to produce response suppression relative to pre-trial sway, presumably as a component of an alerting or orienting response. The fact that this suppression was greatest in the control group indicates that a UCS must overcome this suppression before a well defined response can occur.

In conditioning work the UCS (and CR) are usually defined and measured relative to a pre-trial or baseline level. Figure 2 suggests that a UCS of .2 or $.5 \mathrm{cps}$ of high amplitude should be optimal for conditioning experiments since groups receiving these combinations of frequency and amplitude showed the most clearly differentiated responses to the eliciting stimulus.

We conclude that mild electrical stimulation of the vestibular apparatus elicits slight changes in equilibrium and causes bodily sway. With excessive vestibular stimulation, however, sway becomes inhibited, presumably by way of somatic and proprioceptive feedback mechanisms which compensate for disturbances of equilibrium. This experiment might guide future investigators in selecting intensity and frequency values of the electrical stimulus. The optimal stimulus values selected will be different for conditioning work than for studies of overall postural sway.

\section{References}

Dzendolet, E. Sinusoidal electrical stimulation of the human vestibular apparatus. Percept. mot. Skills, 1963, 17, 171-185.

Revusky, B. T. L., Moore, J. W., \& Dzendolet, E. Conditioning of the human vestibular sway response. Percept. mot. Skills, 1965. 20, 593-600.

\section{Notes}

1. Now at Indiana University.

2. Significance levels for the difference between any two means plotted in Fig. I were obtained from 1 tests based upon the "error" mean square for the third order interaction from analysis of variance. This UCS frequency by UCS amplitude by Score interval (pre- vs, on-trial) by Trial type (conditioning vs. test trial) interaction was significant $(p<.025)$. Differences between means greater than $16.7 \mu \mathrm{v}$ and $22.2 \mu \mathrm{v}$ are significant at the .05 and .01 levels, respectively. 